\title{
Dynamics of flagellar force generated by a hyperactivated spermatozoon
}

\author{
Sumio Ishijima \\ Department of Bioengineering, Graduate School of Bioscience and Biotechnology, Tokyo Institute of Technology, \\ Tokyo 152-8551, Japan \\ Correspondence should be addressed to S Ishijima; Email: sishijim@bio.titech.ac.jp
}

\begin{abstract}
The flagellar force generated by a hyperactivated monkey spermatozoon was evaluated using the resistive force theory applied to the activated (nonhyperactivated) and hyperactivated flagellar waves that were obtained using high-speed video microscopy and digital image processing in order to clarify the mechanism of sperm penetration through the zona pellucida. No difference in the maximum propulsive force, which was parallel to the longitudinal sperm head axis, was found between the activated and hyperactivated spermatozoa. The maximum transverse force $(45 \mathrm{pN})$, which was perpendicular to the longitudinal sperm head axis, of the hyperactivated spermatozoon was $\sim 2.5$ times its propulsive force. As the beat frequency of the flagellar beating remarkably decreased during the hyperactivation, the slowly oscillating transverse force $(5 \mathrm{~Hz})$ by the hyperactivated spermatozoon seems to be most effective for sperm penetration through the zona pellucida.

Reproduction (2011) 142 409-415
\end{abstract}

\section{Introduction}

It is well known that the characteristic movement of the hyperactivated spermatozoa is effective for sperm penetration through the zona pellucida (Boatman \& Robbins 1991, Yanagimachi 1994). This effect has been ascribed to the large thrust (the propulsive force) generated by the hyperactivated spermatozoon (Drobnis et al. 1988, Yanagimachi 1994). However, the role of the thrust generated by the spermatozoon during the zona penetration is controversial (Cherr \& Drobnis 1991, Green 2002) because of lack of rigorous examinations.

Detailed analyses of the movements of the hyperactivated spermatozoon revealed that the large lateral displacements and slow oscillations of the sperm head and flagellum were inherent features of the hyperactivated spermatozoon (Ishijima et al. 2002). These features resulted from the sharp bends in the proximal midpiece and a low beat frequency of the hyperactivated spermatozoon (Ohmuro \& Ishijima 2006). These results suggest that not the propulsive thrust (the propulsive force) but the transverse force generated by a slowly oscillating hyperactivated spermatozoon is an essential feature and plays an important role in sperm penetration through the zona pellucida.

The hydrodynamic calculation of power output in the hyperactivated spermatozoon indicated that the power output did not change during the hyperactivation (Katz et al. 1978). Furthermore, recent detailed analyses of flagellar movements of a spermatozoon using digital image processing revealed that the rate of microtubule sliding between doublet microtubules in the sperm flagella remained constant during the hyperactivation (Ishijima 2007), suggesting that the ATP concentration in the spermatozoon remains constant because the sliding velocity of the doublet microtubules is closely related to the ATP concentration (Gibbons \& Gibbons 1972). These results also suggest that the propulsive thrust generated by a spermatozoon does not increase during the hyperactivation.

To resolve this apparent dispute about the flagellar force and understand the mechanisms by which the sharp bends in the proximal midpiece and slow beatings of the hyperactivated spermatozoon enable sperm to penetrate through zona pellucida, it is necessary to clarify the dynamics of the flagellar force of the hyperactivated spermatozoon using the flagellar waves that are obtained by high-speed video microscopy and digital image analysis. Rigorous analysis of highly curved flagellar waves, such as those of the hyperactivated spermatozoon, is complicated (Gray 1958) and hence has not yet been carried out in this subject.

In this study, the hydrodynamic calculation of the flagellar force of the activated (nonhyperactivated) and hyperactivated spermatozoa has been carried out by applying resistive force theory to the flagellar waves that are obtained from the monkey spermatozoon. 
The maximum propulsive force, which is parallel to the axis of the sperm head, did not increase during the hyperactivation, whereas the maximum transverse force, perpendicular to the axis of the sperm head, was more than double the propulsive force in the hyperactivated spermatozoon. Based on these results, the mechanisms by which the sperm penetrate the zona pellucida are proposed.

\section{Results}

Typical examples of swimming trajectories of activated and hyperactivated spermatozoa are shown in Fig. 1. The heads of the activated spermatozoa moved in nearly straight paths (Fig. 1A), suggesting that mainly propulsive force was effective. However, the heads of the hyperactivated spermatozoa moved in small circular or figure-of-eight paths (Fig. 1B), suggesting that the flagellar force had the transverse component larger than the propulsive force.

Detailed analysis of the flagellar force generated by the hyperactivated spermatozoa was carried out and its result was compared with that of the activated spermatozoa. Typical time courses of the flagellar forces during a complete beat cycle are shown for the activated (Fig. 2A) and hyperactivated (Fig. 3A) spermatozoa. The propulsive force altered its direction twice during each beat cycle, whereas the transverse force altered once (Gray 1953, 1958, Dresdner et al. 1980). The propulsive force generated by the activated spermatozoon repeated at intervals of $\sim 20 \mathrm{~ms}$ (Fig. 2A); therefore, it changed direction $\sim 50$ times per second. However, the transverse force of the activated spermatozoon repeated at intervals of $\sim 40 \mathrm{~ms}$; thus, it changed direction $\sim 25$ times per second (Fig. 2A), a value that corresponds to the beat frequency of the activated spermatozoon (24 Hz, Ishijima et al. 2006). However, the propulsive force of the hyperactivated spermatozoon repeated at intervals of $\sim 100 \mathrm{~ms}$ (Fig. 3A); thus, it changed direction $\sim 10$ times per second, but in contrast, the transverse
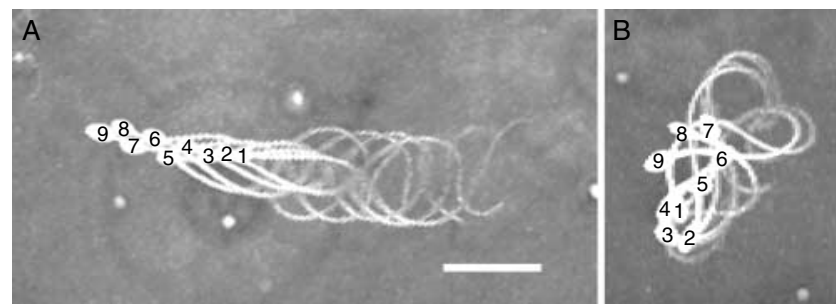

Figure 1 Superimposed images of sperm movements of the activated and hyperactivated monkey spermatozoa. Activated (A) and hyperactivated (B) spermatozoa. The numbers in the center of the sperm heads indicate successive positions of the sperm heads at $20 \mathrm{~ms}$ intervals. The entire length of a flagellum is not always focused. Therefore, the flagellar beating is three dimensional, but the deviation from the beating plane is not so large because the depth of focus of $40 \times$ objective is $\sim 0.65 \mu \mathrm{m}$. Scale bar, $20 \mu \mathrm{m}$.
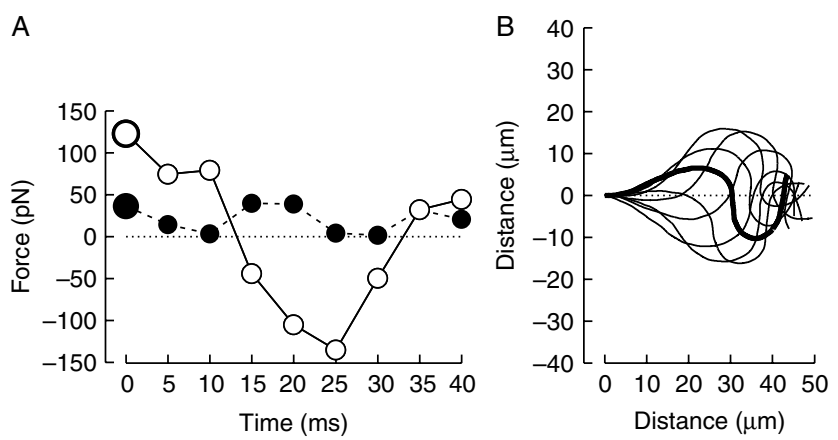

Figure 2 The flagellar forces of the activated monkey spermatozoon. Changes in the flagellar forces (A, propulsive force (filled circle) and transverse force (open circle)) and the corresponding flagellar waves (B) are shown during a complete beat cycle. The flagellar waves are shown at $5 \mathrm{~ms}$ intervals. The large circles at zero time in A correspond to the thick curves in $\mathrm{B}$. The propulsive force directed from the flagellum to the head is taken as positive.

force of the hyperactivated spermatozoon repeated at intervals of $\sim 200 \mathrm{~ms}$; thus, it changed direction $\sim 5$ times per second (Fig. 3A), a value that also corresponds to the beat frequency of the hyperactivated spermatozoon $(4.1 \mathrm{~Hz}$, Ishijima et al. 2006). These results indicated that the propulsive force of the activated spermatozoon oscillated at very high frequencies $(50 \mathrm{~Hz})$, whereas the transverse force of the hyperactivated spermatozoon slowly oscillated $(5 \mathrm{~Hz})$.

The mean values of the maximum propulsive and transverse forces are shown in Table 1. During the hyperactivation, the maximum propulsive force did not change, but the maximum transverse force significantly decreased, namely, the flagellar force did not increase during the hyperactivation. However, the maximum transverse force of the hyperactivated spermatozoon was 2.5 times its maximum propulsive force, suggesting that not the propulsive force but the transverse force is essential for the sperm hyperactivation. Furthermore, in the case of the hyperactivated spermatozoon that had relatively symmetrical flagellar waves, the transverse force of $\sim 45 \mathrm{pN}$ (Table 1) was exerted on both sides because the sperm flagellum oscillated from side to side to almost the same extent (Fig. 3). The flagellar waves producing the maximum and minimum forces are shown in Figs $2 \mathrm{~B}$ and $3 \mathrm{~B}$. The maximum and minimum propulsive forces were obtained from the flagellar waves in which the large basal bends propagated toward the tip of the flagellum, whereas the maximum and minimum transverse forces were obtained from the flagellar waves having sharp basal bends in the midpiece of the flagellum. These features appeared in both the activated and the hyperactivated spermatozoa, although their force values were different. The activated sperm flagellum contained at least a full wave (Fig. 2B), whereas an almost half wave was present in the hyperactivated sperm flagellum (Fig. 3B); thus, the resultant transverse force of the 
A
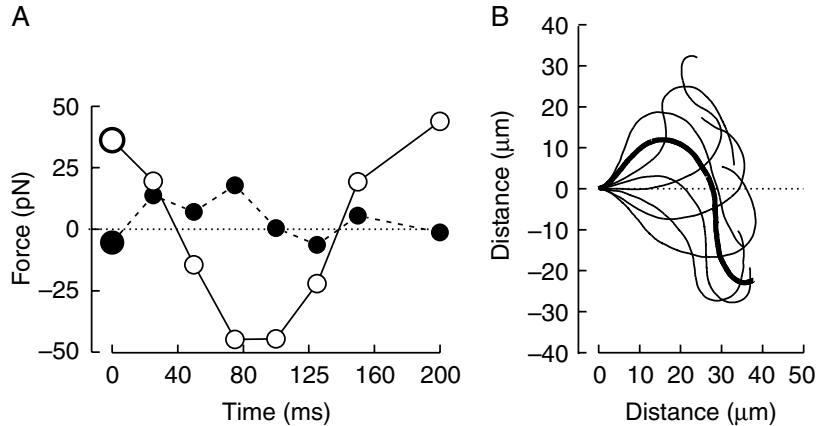

Figure 3 The flagellar forces of the hyperactivated monkey spermatozoon. Changes in the flagellar forces (A, propulsive force (filled circle) and transverse force (open circle)) and the corresponding flagellar waves (B) are shown during a complete beat cycle. The flagellar waves are shown at $29 \mathrm{~ms}$ intervals. The large circles at zero time in A correspond to the thick curves in B. The propulsive force directed from the flagellum to the head is taken as positive.

whole cell was almost balanced on the activated sperm flagellum but was little balanced on the hyperactivated sperm flagellum. This difference in the flagellar wave number partly caused the difference in the maximum and minimum values of the transverse force between the activated and the hyperactivated spermatozoon (Gray 1958, Machemer 1974). The mean propulsive force of the activated spermatozoon was positive (Fig. 2A); thus, this force continuously propels the spermatozoon forward during free swimming (Fig. 1A). However, in the hyperactivated spermatozoon, the difference between the maximum and the minimum propulsive forces alternately changed its sign, and accordingly, the nonprogressive movement occurred during the free swimming (Fig. 1B).

The flagellar forces exerted by a flagellar segment are proportional to its velocities of the longitudinal and transverse displacements and its shear angle (Materials and Methods). The contribution of these factors to the flagellar force of the activated and hyperactivated spermatozoa was estimated using equation 1 (Materials and Methods). Because the change in the flagellar force caused by different shear angles was half its velocity, the flagellar force generated by its segment is roughly able to be estimated by its velocity (Fig. 4). Therefore, the maximum transverse force by a flagellar segment of the activated spermatozoon was obtained when the flagellar segment was crossing the sperm head axis, whereas the maximum propulsive force was obtained when the flagellar segment was near its maximum transverse displacement. However, in the hyperactivated spermatozoa, both the maximum transverse and propulsive forces were obtained when the flagellar segment was crossing the sperm head axis because the maximum propulsive and transverse velocities were obtained at this position (Fig. 4).

\section{Discussion}

\section{Flagellar force of a sperm flagellum}

Calculation of the flagellar force using the resistive force theory was accurate and reliable for the sea urchin spermatozoon (Gray \& Hancock 1955). However, it has not yet established whether this theory is applicable to other types of flagellar movements because there are only a few reports comparing the calculated data with the experimental values. Therefore, in order to estimate the calculation error of the flagellar force using the resistive force theory, the values obtained by analytical solution of the triangular waves were compared to those obtained using the resistive force theory. The calculation error was within $13 \%$ and negligibly small compared with the experimental error (Ishijima et al. 2006).

There have been several studies calculating and measuring the flagellar force generated by a spermatozoon other than a sea urchin spermatozoon (Baltz et al. 1988, König et al. 1996). The calculated propulsive thrust was $19 \mathrm{pN}$ for the human spermatozoon (Baltz et al. 1988); thus, $\sim 18 \mathrm{pN}$ of the propulsive force for monkey spermatozoon in this study are reasonable because its characteristics of the morphology and the flagellar movement are almost same (Cummins \& Woodall 1985, Ishijima et al. 2006). Experimental measurement of the flagellar force using optical tweezers gave a mean value of $44 \mathrm{pN}$ for the human spermatozoon (König et al. 1996).

In this study, the propulsive force (the propulsive thrust) did not increase during the hyperactivation. Therefore, the zona penetration by the propulsive force of the hyperactivated spermatozoon is improbable even though the oscillation frequency of the propulsive force decreased from 50 to $10 \mathrm{~Hz}$. However, the transverse force of the hyperactivated spermatozoon was 2.5 times the propulsive force. Furthermore, the transverse force nearly doubled itself during the symmetrical flagellar beating of the hyperactivated spermatozoon, namely, the symmetrical flagellar waves generated $45 \mathrm{pN}$ in both directions due to their side-to-side oscillation. All hyperactivated spermatozoa did not produce

Table 1 The maximum flagellar forces produced by a monkey spermatozoon $(\mathrm{pN})$.

\begin{tabular}{lcr}
\hline & $\begin{array}{c}\text { Propulsive force } \\
(n, N)\end{array}$ & $\begin{array}{c}\text { Transverse force }^{\mathrm{a}} \\
(n, N)\end{array}$ \\
\hline Activated sperm & $17.4 \pm 2.7(12,3)^{*}$ & $128.9 \pm 24.9(12,3)$ \\
Hyperactivated sperm & $18.3 \pm 6.5(13,3)^{*}$ & $44.9 \pm 11.5(13,3)$ \\
\hline
\end{tabular}

Data are presented as mean \pm s.D. $N$, number of monkeys used for the experiments; $n$, number of measured spermatozoa. *No significant difference $(P<0.01)$.

${ }^{a}$ The propulsive force is a component of the flagellar force in the direction longitudinal to the sperm head, whereas the transverse force is a component of the flagellar force in the direction transverse to the sperm head axis. 

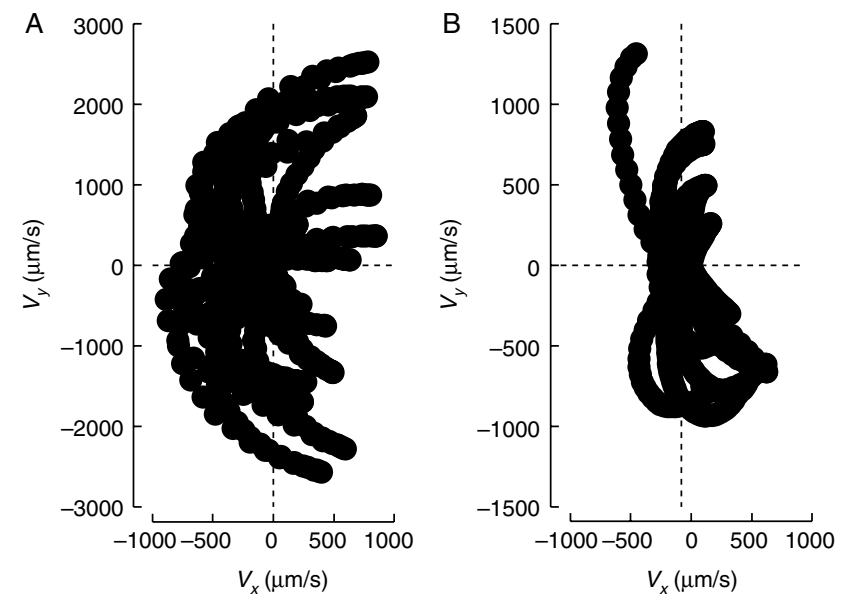

Figure 4 The change in the velocity of the flagellar segments of the activated and the hyperactivated spermatozoa. The propulsive $\left(V_{x}\right)$ and transverse $\left(V_{y}\right)$ velocities of the flagellar segments are shown during a single beat cycle for the activated $(\mathrm{A})$ and hyperactivated $(\mathrm{B})$ spermatozoa shown in Figs 2B and 3B.

symmetrical flagellar waves, but the percentage of the hyperactivated spermatozoa producing symmetrical flagellar waves increased during incubation (Yanagimachi 1994, Ishijima et al. 2006). Almost all reported studies have overlooked or ignored the symmetrical flagellar waves of the hyperactivated spermatozoon (Ho et al. 2002, Suarez 2008); however, the advantages of the symmetrical flagellar waves in generating the transverse force are probably an important feature of the hyperactivation. These results suggest that most important features of the hyperactivation are the transverse force higher than the propulsive force and the low beat frequency (below).

\section{Mechanisms of sperm penetration through the zona pellucida}

The low beat frequency in the hyperactivated spermatozoon resulted from its sharp bends of the sperm flagellum because the spermatozoon did not change the energy consumption during the hyperactivation (Ohmuro \& Ishijima 2006, Ishijima 2007, Kaneko et al. 2007). As seen in Table 1, the flagellar forces of the activated spermatozoon are not as low as those of the hyperactivated spermatozoon, suggesting that not the large flagellar waves but the low beat frequency is the most important feature of the hyperactivation. Several recent studies examining the physical property of zona pellucida and living cells seemed to determine the true meaning of this feature of the hyperactivation, namely, the zona pellucida showed the transition from elastic behavior to plastic depending on the deformation (Wacogne et al. 2008, Papi et al. 2009), and such a large deformation of the cell surface was due to the lowfrequency oscillations (Fabry et al. 2003). Taking into account all these results, it is likely that the slow oscillation of the hyperactivated spermatozoon fully exploits the plastic behavior of the zona pellucida, namely, the slowly oscillating shear force generated by the hyperactivated sperm flagellum causes a large deformation in the zona material due to the rearrangement of the zona structure. In fact, many biological materials show biphasic features: predominantly viscous at low frequencies and predominantly elastic at high frequencies (Balazs \& Gibbs 1970). Consequently, this must be the main reason why the activated spermatozoon beating with high frequencies fails to attach and penetrate the zona pellucida even though it has a higher flagellar force than the hyperactivated spermatozoon. Furthermore, repetitive shear stress causes a large deformation at a lower shear force than that required, thus causing deformation in a single application (Suresh 1998).

The propulsive force is necessary for the sperm penetration through the zona pellucida regardless of whether the acrosomal enzymes are involved, otherwise the zona pellucida pulls the sperm into the perivitelline space. However, this study revealed that the propulsive force was lower than the transverse force, suggesting that the transverse force plays a crucial role in the sperm penetration through the zona pellucida. This idea is also supported by the several observations, namely, the sperm head laterally oscillated within the zona pellucida (Yanagimachi 1966, Bedford 1991). Furthermore, several recent studies examining the movement of the micropipette tip during the zona pellucida piercing in piezo-assisted ICSI revealed that not the longitudinal displacement of the micropipette tip but its transverse displacement was critical in piercing the zona pellucida (Ediz \& Olgac 2004, 2005). Because the piezo-assisted ICSI resulted in a higher efficiency of microinjection even with a flat tip micropipette than the conventional ICSI in which the micropipette with a sharpened tip was rapidly pushed forward until it penetrated the zona pellucida (Kimura \& Yanagimachi 1995, Yanagida et al. 1998), the shear force generated by the lateral movement of the micropipette is probably more effective in piercing the zona pellucida than the propulsive thrust.

As mentioned above, the sperm penetration through the zona pellucida must be mainly mechanical. However, during sperm binding and initial entry into the zona pellucida, acrosomal enzymes must play an important role. As seen in Fig. 3A, the propulsive force of the hyperactivated spermatozoon oscillated back and forth and had the maximum and minimum values due to the longitudinal displacement of the flagellar segments; thus, the sperm head not only pushes the zona surface but also alternately leaves it. It might be the acrosomal enzymes that fuse the sperm head and the zona surface to prevent the sperm from leaving the zona surface (Tollner et al. 2003). In summary, the hyperactivated 
spermatozoon seems to penetrate through the zona pellucida by the shear force generated by the slow oscillation of the transverse force while pushing the zona surface by the propulsive force.

\section{Materials and Methods}

\section{Sperm preparations}

The spermatozoa obtained from adult male cynomolgus macaques, which were caged individually at the California Regional Primate Research Center in compliance with the Federal Animal Welfare Act and the National Institutes of Health Guidelines for the Care and Use of Laboratory Animals, were prepared as described by Tollner et al. (2003). Briefly, the semen samples collected by electro-ejaculation into $15 \mathrm{ml}$ centrifuge tubes containing Hepes-buffered BWW were allowed to stand for $30 \mathrm{~min}$ to remove the coagulum, then further diluted with Hepes-buffered BWW containing $3 \mathrm{mg} / \mathrm{ml} \mathrm{BSA}$, and washed by centrifugation at $300 \mathrm{~g}$ for $10 \mathrm{~min}$. The resulting sperm pellets were centrifuged through a $6 \mathrm{~cm}$ column of $80 \%$ Percoll at $400 \mathrm{~g}$ for $25 \mathrm{~min}$. The supernatant containing Percoll and any remaining seminal plasma was removed and then the sperm pellet was resuspended in bicarbonate-buffered BWW containing $30 \mathrm{mg} / \mathrm{ml}$ BSA. The obtained sperm suspensions were washed two more times by centrifugation at $300 \mathrm{~g}$ for $10 \mathrm{~min}$ and diluted in this medium.

The spermatozoa were finally resuspended at a concentration of $10-20 \times 10^{6} / \mathrm{ml}$ in the bicarbonate-buffered BWW and then capacitated by a series of incubations beginning with a $24 \mathrm{~h}$ incubation at room temperature in $4.5 \% \mathrm{CO}_{2}$. Following this room temperature incubation, the sperm suspensions were incubated at $37^{\circ} \mathrm{C}$ in $4.5 \% \mathrm{CO}_{2}$ for $2 \mathrm{~h}$ more, at which point the sperm concentration was adjusted to $10 \times 10^{6} / \mathrm{ml}$. The sperm suspensions were incubated for an additional $1 \mathrm{~h}$ in media containing $1 \mathrm{mM}$ caffeine and $1 \mathrm{mM} \mathrm{dbcAMP}$ (as activators). Macaque sperm capacitated according to this protocol have been used for in vitro fertilization studies and produce high rates of fertilization after a $4 \mathrm{~h}$ coincubation of sperm and oocytes. All procedures were performed at room temperature $\left(23^{\circ} \mathrm{C}\right)$ unless noted.

\section{Observations and recording}

For examination and recording of the movements of the sperm and their flagella, a $70 \mu \mathrm{l}$ aliquot of the sperm suspension was transferred to a $20 \mu \mathrm{m}$ deep observation chamber that was made from four posts of silicon grease containing 5-20 $\mu \mathrm{m}$ silica beads and covered with a $22 \times 22 \mathrm{~mm}$ glass coverslip. The slide was warmed for 5 min on a microscope stage warmer set at $37^{\circ} \mathrm{C}$ before the sperm were added. The sperm and their flagellar movements of the monkey spermatozoa were observed and recorded using an Olympus phase-contrast microscope equipped with a condenser, a $\times 40 \mathrm{NH}$ objective, and $\times 10$ eyepieces (Scientific Instruments Co., Sunnyvale, CA, USA). Images were directly captured and stored in a computer using a high-speed camera (HAS-200R, Ditect Co. Ltd, Tokyo, Japan), image software (Dipp-Motion 2D, Ditect Co. Ltd), and a frame grabber (HAS-PCl, Ditect Co. Ltd) at the rate of 200/s for about $2 \mathrm{~s}$.

\section{Calculation of flagellar force}

For the detailed field-by-field analysis, images of the beating flagellum of the monkey spermatozoon attached to the slide surface by its head were analyzed using image analysis software (Bohboh, Bohboh Soft, Tokyo, Japan; Baba \& Mogami 1985, Ishijima et al. 2002, 2006, Ohmuro \& Ishijima 2006) in order to simulate the spermatozoa attached to the zona pellucida. An individual flagellar image was automatically tracked using the Autotrace module of the Bohboh Software; the coordinate values of the flagellum from the head-midpiece junction to the flagellar tip were obtained with reference to the direction of the axis of the sperm head. The shear angle, which is the angle of the tangent to the flagellum with reference to the sperm head axis, was calculated from the coordinate values obtained as a function of the distance along the flagellum from the head-midpiece junction. The beat frequency was calculated from the period required for a complete beating cycle.

Monkey spermatozoa incubated in capacitation medium exhibited two distinct swimming patterns: $\sim 30 \%$ of spermatozoa swam in nearly straight paths with high values of beat frequency and the remainder was characterized by large lateral displacement of the head and low values of beat frequency (Ishijima et al. 2006). We refer to the former movement as activated (nonhyperactivated) and the latter movement as hyperactivated (Boatman \& Bavister 1984, Tollner et al. 2003, Ishijima et al. 2006). Movement characteristics of the hyperactivated spermatozoa were fundamentally different from those of the activated ones, namely, the beat frequency of flagellar movement $(24 \mathrm{~Hz})$ decreased approximately to one-sixth $(4.1 \mathrm{~Hz})$ and the curvature of flagellar bends at the base of the midpiece $(0.065 / \mu \mathrm{m})$ increased approximately to four times $(0.25 / \mu \mathrm{m})$ during the hyperactivation (Ishijima et al. 2006). The flagellar forces generated by the essentially different movements of the activated and hyperactivated spermatozoa were quantitatively estimated and compared with each other.

The flagellar force was calculated using the resistive force theory (Gray 1958) and the drag coefficient correction by Lighthill (1976). The flagellum was considered to be a series of cylindrical segments; each segment independently interacted with the medium. The force generated by each segment is the product of the drag coefficient, its velocity, and its length. Therefore, the propulsive force $(d \mathrm{~F})$ and the transverse force $(d T)$ exerted by an inclined segment at any instant are defined as follows:

$$
\begin{aligned}
& d \mathrm{~F}=\left(V_{x}\left(C_{\mathrm{N}} \sin ^{2} \theta+C_{\mathrm{L}} \cos ^{2} \theta\right)+V_{y}\left(C_{\mathrm{N}}-C_{\mathrm{L}}\right) \sin \theta \cos \theta\right) d s, \\
& d \mathrm{~T}=\left(V_{x}\left(C_{\mathrm{N}}-C_{\mathrm{L}}\right) \sin \theta \cos \theta+V_{y}\left(C_{\mathrm{N}} \cos ^{2} \theta+C_{\mathrm{L}} \sin ^{2} \theta\right)\right) d s,
\end{aligned}
$$

where $C_{\mathrm{N}}$ and $C_{\mathrm{L}}$ are the drag coefficients for movement normal and tangential to the segment, respectively, $\theta$ is the shear angle, and $d s$ is the segment length. According to Lighthill (1976), $C_{\mathrm{N}}$ and $C_{\mathrm{L}}$ of thin fibers such as a flagellum are obtained by

$C_{N}=4 \pi \eta /(1 n(2 q / a)+0.5)$,

$C_{\mathrm{L}}=2 \pi \eta / 1 n(2 q / a)$, 
where $\eta$ is the viscosity of the medium, $q=0.09 \Lambda$ (where $\Lambda$ is the wavelength measured along the flagellum), and a is the radius of the flagellum. Substituting the values of $\eta=0.7 \times 10^{-3}$ Pa.s, $\Lambda=50 \mu \mathrm{m}$ for the activated spermatozoon and $100 \mu \mathrm{m}$ for the hyperactivated spermatozoon (Ishijima et al. 2006), and the average radius of the flagellum, $\mathrm{a}=0.317 \mu \mathrm{m}$, gave values of $C_{\mathrm{N}}=1.31 \times 10^{-3}$ Pa.s and $C_{\mathrm{L}}=$ $2.29 \times 10^{-3}$ Pa.s for the activated spermatozoon and $C_{\mathrm{N}}=$ $1.94 \times 10^{-3}$ Pa.s and $C_{\mathrm{L}}=1.09 \times 10^{-3}$ Pa.s for the hyperactivated spermatozoon. The drag coefficients were not adjusted for the radius of the flagellum because the flagellar force differed by $<4 \%$. The velocities of displacement longitudinal $\left(V_{x}\right)$ and transverse $\left(V_{y}\right)$ to the sperm head axis are as follows:

$V_{x}=\left(x_{i+1}-x_{i}\right) k$,

$V_{y}=\left(y_{i+1}-y_{i}\right) k$,

where $x$ is the $x$-coordinate, $y$ is the $y$-coordinate, $i$ is the frame number, and $k$ is the frame rate $(\mathrm{Hz})$. The total propulsive and transverse forces generated by a flagellum were obtained by numerically integrating $d \mathrm{~F}$ and $d \mathrm{~T}$ from the head-midpiece junction to the flagellar tip. By eliminating the shear angle from the equations of the propulsive $(d F)$ and transverse $(d T)$ forces, the relationship between the flagellar forces and the velocities was obtained as follows:

$\left(d \mathrm{~F} / C_{\mathrm{L}} / d s-3 / 2 V_{x}\right)^{2}+\left(d \mathrm{~T} / C_{\mathrm{L}} / d s-3 / 2 V_{y}\right)^{2}=(V / 2)^{2}$,

where $V=\left(V_{x}^{2}+V_{y}^{2}\right)^{1 / 2}$. This equation indicates that the change in the flagellar force caused by different shear angles is half its velocity.

The calculation error of the flagellar force was within 13\%, which was estimated by comparing the values obtained by analytical solution of the triangular waves to those obtained using the resistive force theory.

\section{Statistical analysis}

All data are expressed as the mean \pm s.D., and any statistical significances were calculated using Student's $t$-test by SPSS 11.0J (SPSS Japan, Inc., Tokyo, Japan). The significant level was considered to be $P<0.01$.

\section{Declaration of interest}

The authors declare that there is no conflict of interest that could be perceived as prejudicing the impartiality of the research reported.

\section{Funding}

This research did not receive any specific grant from any funding agency in the public, commercial or not-for-profit sector.

\section{Acknowledgements}

We thank Drs A I Yudin and T L Tollner for technical assistance.

\section{References}

Baba SA \& Mogami Y 1985 An approach to digital image analysis of bending shapes of eukaryotic flagella and cilia. Cell Motility and the Cytoskeleton 5 475-489. (doi:10.1002/cm.970050605)

Balazs EA \& Gibbs DA 1970 The rheological properties and biological function of hyaluronic acid. In Chemistry and Molecular Biology of The Intercellular Matrix, pp 1241-1253. Ed. EA Balazs. New York: Academic Press.

Baltz JM, Katz DF \& Cone RA 1988 Mechanics of sperm-egg interaction at the zona pellucida. Biophysical Journal 54 643-654. (doi:10.1016/ S0006-3495(88)83000-5)

Bedford JM 1991 The coevolution of mammalian games. In A Comparative Overview of Mammalian Fertilization, pp 3-35. Eds BS Dunbar \& MG O'Rand. New York: Plenum Press.

Boatman DE \& Bavister BD 1984 Stimulation of rhesus monkey sperm capacitation by cyclic nucleotide mediators. Journal of Reproduction and Fertility 71 357-366. (doi:10.1530/jrf.0.0710357)

Boatman DE \& Robbins RT 1991 Bicarbonate: carbon-dioxide regulation of sperm capacitation, hyperactivated motility, and the acrosome reaction. Biology of Reproduction 44 806-813. (doi:10.1095/biolreprod44.5.806)

Cherr GN \& Drobnis EZ 1991 Fertilization in the golden hamster. In A Comparative Overview of Mammalian Fertilization, pp 217-243. Eds BS Dunbar \& MG O'Rand. New York: Plenum Press.

Cummins JM \& Woodall PF 1985 On mammalian sperm dimensions. Journal of Reproduction and Fertility 75 153-175. (doi:10.1530/jrf.0. 0750153)

Dresdner RD, Katz DF \& Berger SA 1980 The propulsion by large amplitude waves of uniflagellar micro-organisms of finite length. Journal of Fluid Mechanics 97 591-621. (doi:10.1017/S0022112080002716)

Drobnis EZ, Yudin Al, Cherr GN \& Katz DF 1988 Hamster sperm penetration of the zona pellucida: kinematic analysis and mechanical implications. Developmental Biology 130 311-323. (doi:10.1016/00121606(88)90437-X)

Ediz K \& Olgac N 2004 Microdynamics of the piezo-driven pipettes in ICSI. IEEE Transactions on Bio-Medical Engineering 51 1262-1268. (doi:10. 1109/TBME.2004.827346)

Ediz K \& Olgac N 2005 Effect of mercury column on the microdynamics of the piezo-driven pipettes. Journal of Biomechanical Engineering 127 531-535. (doi:10.1115/1.1894368)

Fabry B, Maksym GN, Butler JP, Glogauer M, Navajas D, Taback NA, Millet EJ \& Fredberg JJ 2003 Time scale and other invariants of integrative mechanical behavior in living cells. Physical Review E, Statistical, Nonlinear, and Soft Matter Physics 68 041914. (doi:10.1103/PhysRevE. 68.041914)

Gibbons BH \& Gibbons IR 1972 Flagellar movement and adenosine triphosphate activity in sea urchin sperm extracted with Triton X-100. Journal of Cell Biology 54 75-97. (doi:10.1083/jcb.54.1.75)

Gray J 1953 Undulatory propulsion. Quarterly Journal of Microscopical Science 94 551-578.

Gray J 1958 The movement of the spermatozoa of the bull. Journal of Experimental Biology 35 96-108.

Gray J \& Hancock JG 1955 The propulsion of sea-urchin spermatozoa. Journal of Experimental Biology 32 802-814.

Green DPL 2002 Fertilization biophysics. In Fertilization, pp 387-399. Ed. DM Hardy. San Diego: Academic Press.

Ho H-C, Granish KA \& Suarez SS 2002 Hyperactivated motility of bull sperm is triggered at the axoneme by $\mathrm{Ca}^{2+}$ and not cAMP. Developmental Biology 250 208-217. (doi:10.1006/dbio.2002.0797)

Ishijima S 2007 The velocity of microtubule sliding: its stability and load dependency. Cell Motility and the Cytoskeleton 64 809-813. (doi:10. 1002/cm.20228)

Ishijima S, Baba SA, Mohri H \& Suarez S 2002 Quantitative analysis of flagellar movement in hyperactivated and acrosome-reactivated golden hamster spermatozoa. Molecular Reproduction and Development 61 376-384. (doi:10.1002/mrd.10017)

Ishijima S, Mohri H, Overstreet JW \& Yudin Al 2006 Hyperactivation of monkey spermatozoa is triggered by $\mathrm{Ca}^{2+}$ and completed by cAMP. Molecular Reproduction and Development 73 1129-1139. (doi:10. 1002/mrd.20420) 
Kaneko T, Mōri T \& Ishijima S 2007 Digital image analysis of the flagellar beat of activated and hyperactivated Suncus spermatozoa. Molecular Reproduction and Development 74 478-485. (doi:10.1002/mrd.20630)

Katz DF, Yanagimachi R \& Dresdner RD 1978 Movement characteristics and power output of guinea-pig and hamster spermatozoa in relation to activation. Journal of Reproduction and Fertility 52 167-172. (doi:10. 1530/jrf.0.0520167)

Kimura Y \& Yanagimachi R 1995 Intracytoplasmic sperm injection in the mouse. Biology of Reproduction 52 709-720. (doi:10.1095/biolreprod52.4.709)

König K, Svaasand L, Liu Y, Sonek G, Patrizio P, Tadir Y, Berns MW \& Tromberg BJ 1996 Determination of motility forces of human spermatozoa using an $800 \mathrm{~nm}$ optical trap. Cellular and Molecular Biology 42 501-509.

Lighthill J 1976 Flagellar hydrodynamics. SIAM Review 18 161-230. (doi:10.1137/1018040)

Machemer H 1974 Ciliary activity and metachroism in protozoa. In Cilia and Flagella, pp 199-286. Ed. MA Sleigh. London: Academic Press.

Ohmuro J \& Ishijima S 2006 Hyperactivation is the mode conversion from constant-curvature beating to constant-frequency beating under a constant rate of microtubule sliding. Molecular Reproduction and Development 73 1412-1421. (doi:10.1002/mrd.20521)

Papi M, Sylla L, Parasassi T, Brunelli R, Monaci M, Maulucci G, Missori M, Arcovito G, Ursini F \& Spirito MD 2009 Evidence of elastics to plastic transition in the zona pellucida of oocytes using atomic force spectroscopy. Applied Physics Letters 94 153902. (doi:10.1063/1.3107265)

Suarez SS 2008 Control of hyperactivation in sperm. Human Reproduction Update 14 647-657. (doi:10.1093/humupd/dmn029)
Suresh S 1998 Cyclic deformation and crack initiation in noncrystalline solids. In Fatigue of Materials, edn 2, ch 6, pp 200-217. Cambridge UK: Cambridge University Press.

Tollner TL, Yudin AI, Cherr GN \& Overstreet JW 2003 Real time observations of individual macaque sperm undergoing tight binding and the acrosome reaction on the zona pellucida. Biology of Reproduction 68 664-672. (doi:10.1095/biolreprod.102.009175)

Wacogne B, Pieralli C, Roux C \& Gharbi T 2008 Measuring the mechanical behavior of human oocytes with a very simple SU-8 micro-tool. Biomedical Microdevices 10 411-419. (doi:10.1007/s10544-007-9150-7)

Yanagida K, Katayose H, Yazawa H, Kimura Y, Konnai K \& Sato A 1998 The usefulness of a piezo-micromanipulator in intracytoplasmic sperm injection in humans. Human Reproduction 14 448-453. (doi:10.1093/ humrep/14.2.448)

Yanagimachi R 1966 Time and process of sperm penetration into hamster ova in vivo and in vitro. Journal of Reproduction and Fertility 11 359-370. (doi:10.1530/jrf.0.0110359)

Yanagimachi R 1994 Mammalian fertilization. In The Physiology of Reproduction, edn 2, pp 189-317. Eds E Knobil \& JD Neill. New York: Raven Press.

Received 28 October 2010

First decision 9 December 2010

Revised manuscript received 19 May 2011

Accepted 13 June 2011 